\title{
ReCollection
}

\section{Dr. Zhen-Yi Wang: Fighting leukemia}

\author{
Ming $\mathrm{Li}^{1}$, Guang-Biao Zhou ${ }^{2}$ \\ ${ }^{1}$ Beijing Institutes of Life Science, Chinese Academy of Sciences, Beijing 100101, China \\ 2 Institute of Zoology, Chinese Academy of Sciences, Beijing 100101, China
}

Dr. Zhen-Yi Wang (Fig. 1), Academician of the Chinese Academy of Engineering, a professor at the Medical School of Shanghai Jiao Tong University and the honorary director of the Shanghai Institute of Hematology, is best known for his contribution in developing a differentiation induction therapy of acute promyelocytic leukemia (APL).

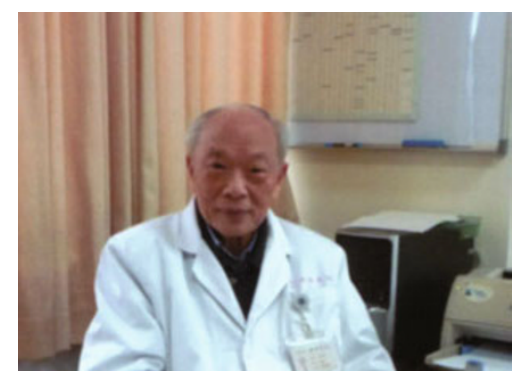

Figure 1. Dr. Zhen-Yi Wang in his office

Graduated with a MD degree from the Aurora University School of Medicine in Shanghai in 1948, Dr. Wang was recruited as a resident doctor by Rui-Jin Hospital. Upon his promotion in 1952 , he was required to choose a field to major in. He chose hematology and it was the beginning of his life-long pursuit. The reason behind his decision was quite unusual-he loved music and in his mind, blood cells were just as simple and beautiful as musical notes when examined under a microscope. However, when he started working in the hematology department, reality finally hit himblood diseases were neither simple nor beautiful; they showed no mercy when taking lives away.
Back in the 1950s, the clinical approaches for leukemia were quite limited in China, not to mention biomedical research for developing new strategies. Never discouraged by the poor research condition, Dr. Wang started a selftraining program by extensively reading books and research articles about leukemia, especially APL. The more he read, the more he asked himself: Was there any way to treat APL other than cytotoxic chemotherapy? Could the "bad" cancer cells be "educated" and "converted" to good cells, instead of being killed by chemo? Later in the 1970s, he found out that he happened to share the same view with Western researchers who raised the induced cancer differentiation concept. This further intrigued him and gave him firm confidence in the screen for differentiation inducers using HL-60 cells and fresh APL cells. A number of compounds were reported to be capable of inducing cancer cell maturation, including butyrate, dimethyl sulfoxide, and 13 cis-retinoic acid (13 cis-RA). Fortunate enough, an isomer of 13 cis-RA, all-trans retinoic acid (ATRA), was available in Shanghai as a skin disease medicine. In $\mathrm{Dr}$. Wang's screen, ATRA strongly induced terminal differentiation of abnormal promyelocytes, and its inducing effects were superior to 13 cis-RA and other candidate compounds. The intriguing data provided main impetus for the clinical trial of ATRA treatment on APL patients.

In 1985, a five-year-old girl who suffered from APL was admitted into
Shanghai Children's Hospital. She had gone through chemotherapy but it did not alleviate her symptoms such as high fever and severe hemorrhage. Her parents practically gave up hope before the doctors told them about the ATRA trial. Wanting to make every possible effort for their daughter, the parents agreed to try ATRA. After orally taking ATRA for three weeks, her high fever was gone and the hemorrhage stopped. She was then treated with ATRA/chemo as a post-remission treatment for a year. The girl is now over 30-year-old and very stable and healthy. This was the very first case of ATRA treatment on an APL patient and the successful case encouraged Dr. Wang and his colleagues to continue the ATRA trial. By 1988, 24 APL patients had been treated with ATRA in Shanghai and 23 out of 24 went into remission after treatment. The significant results were reported in Blood in 1988, and the article has been cited for nearly 2000 times over the years and became one of the most cited papers on cancer research. The dramatic effects of ATRA on APL were also progressively confirmed by other hospitals and hematology centers in Europe and North America.

For the following decade or so, Dr. Wang and his colleagues made painstaking efforts to optimize the ATRA treatment. ATRA/chemo in combination was proven to provide better effects with less drug resistance and reduced relapse compared with ATRA alone. In the mid1990s, Dr. Zhu Chen, a former student of Dr. Wang and currently the Health 
Minister of China, proved the efficacy of Arsenic Trioxide (ATO), a well-known poison used as a traditional Chinese medicine, in treating replased APL patients. Later on, a synergistic combination of ATRA and ATO was tested in a four-year long clinical trial and proven to be more effective than ATRA or ATO alone. The ATRAVATO combination, often referred to as "Shanghai Protocol", has become the standard therapy for APL worldwide, and turned this deadly disease into a highly curable one.

For his achievements in fighting APL, Dr. Wang has received numerous prizes and awards, including the Kettering Prize from the General Motors Cancer Research Foundation USA in 1994, the Prize of Brupbacher from Switzerland in 1997, the Prize for Science from the Simmon Del Ducca Foundation of France in 1998, and the 7th annual Szent-Gyorgyi Prize from the National Foundation for Cancer Research of US in 2012 (shared with Dr. Chen). He also received the 2010 State Supreme Science and Technology Award of China, presented to him by President Jin-Tao Hu.

Even though he is unquestionably the most famous hematologist in China, fame or fortune has never been $\mathrm{Dr}$ Wang's aim in life. Despite all the prizes and award he has won, the medals and certificates of awards can hardly be found in Dr. Wang's office, which is packed with books and journals. Most of the award money was either shared with his colleagues or donated to charity. Dr. Wang has never considered the option of applying for a patent of ATRA treatment on APL, as it would only increase the financial burden of the patients.

The benefit of patients is always $\mathrm{Dr}$ Wang's number one priority. When he decided to conduct the first clinical trial with ATRA in 1985, some friends and coworkers strongly advised him against it, as the trial would be too risky and if anything should go wrong during the trial, his reputation would be ruined. But for him, saving a life outweighed his personal gain and loss. Putting his whole career at stake, he conducted the trial which turned out to be a great success. So in a way, it was his deep sympathy and affections for patients that made the ATRA trial possible and made him a great doctor. After the first paper of ATRA therapy was published in 1988, Dr. Wang put great efforts into promoting ATRA nation-wide and internationally, so that more patients would receive the treatment sooner. When a Japanese doctor, Ryuzo Ohno, initiated a clinical trial of ATRA in Japan in the early 1990s, he realized that it was extremely difficult and time-consuming to import ATRA from China officially. Dr. Wang heard about this and gave Dr. Ohno a whole bottle of ATRA as a personal gift when they met at a meeting. He later sent more ATRA to Japan whenever possible, mostly through Dr. Ohno's friends who were visiting Shanghai. Without Dr. Wang's help, Dr. Ohno and his team could never have carried out the APL trial in Japan so early.

Love and care, that's what Dr. Wang gives to his patients without reservation. In return, he often receives letters and small gifts from patients, some of whom he treated decades ago. "Only a doctor can receive this kind of gratitude and satisfaction," said Dr. Wang.

\section{REFERENCES}

Wang, Z. (2008). Acute promyelocytic leukemia: from highly fatal to highly curable. Blood 111, 2505-2515. 